\title{
Microwave-Assisted Concise Total Syntheses of Quinazolinobenzodiazepine Alkaloids
}

Ji-Feng Liu, " Mira Kaselj, Yuko Isome, Jenny Chapnick, Bailin Zhang, Grace Bi, Daniel Yohannes, Libing Yu, and Carmen M. Baldino

ArQule, Inc., 19 Presidential Way, Woburn, MA 01801, USA

jliu@arqule.com

Table of Contents

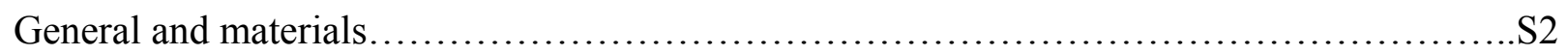

${ }^{1} \mathrm{H}$ and ${ }^{13} \mathrm{C}$ NMR spectra:

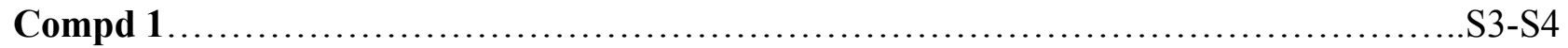

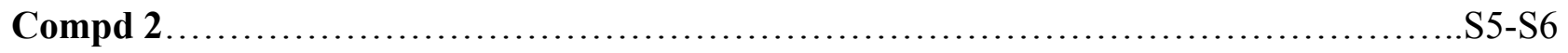

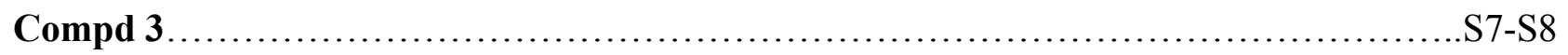

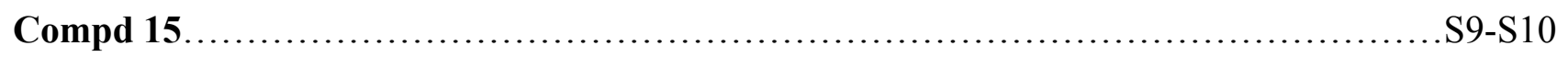

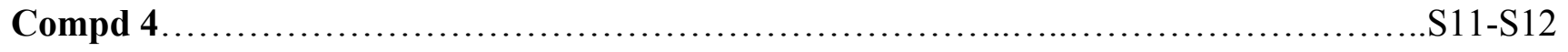

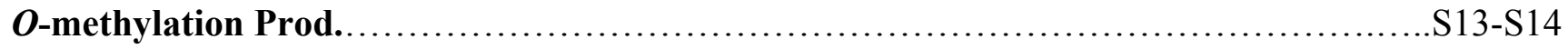

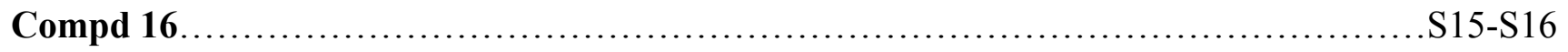

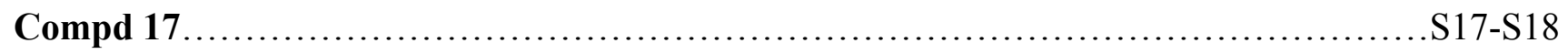




\section{SUPPORTING INFORMATION}

General and materials. All reagents and solvents were purchased and used without further purification. Biotage Smith Synthesizer ${ }^{\mathrm{TM}}$ was used for microwave reactions. Irradiation was initiated at $300 \mathrm{~W}$ to raise the temperature to the set point and then power was applied at intervals and levels to maintain the desired temperature. Reactions were run in sealed vessels. Reaction times reported included time for the vial to ramp to the desired temperature. The product isolation was performed on prep-TLC plates or on preparative HPLC. The Preparative HPLC was carried out employing a ProntoSIL 120-10-C18 column $(50 \times 20 \mathrm{~mm})$. The flow rate was at $44 \mathrm{~mL} / \mathrm{min}$ utilizing an acetonitrile/water mobile phase. ${ }^{1} \mathrm{H}$ NMR (400 MHz) and ${ }^{13} \mathrm{C}$ NMR (100 MHz) spectra were recorded in $\mathrm{CDCl}_{3}$ solution (unless otherwise stated). Chemical shifts are given in parts per million (ppm) downfield from tetramethylsilane (TMS) as an internal reference, and coupling constants ( $J$-values) are in hertz $(\mathrm{Hz})$. Low-resolution mass spectra were recorded in the $\mathrm{ES}^{+}$mode. The high-resolution mass spectra (HRMS) were recorded in the FAB mode. 


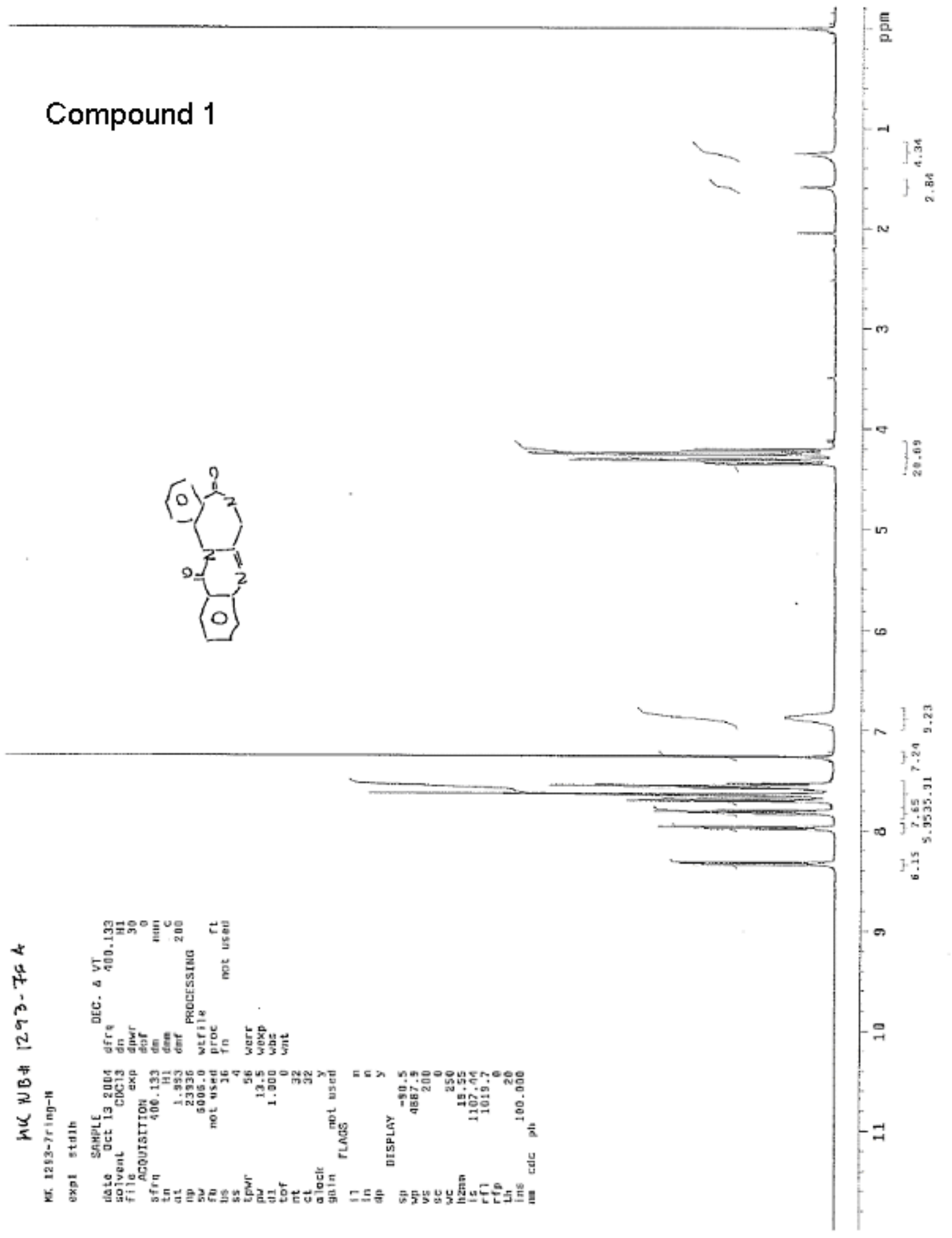




\section{SUPPORTING INFORMATION}

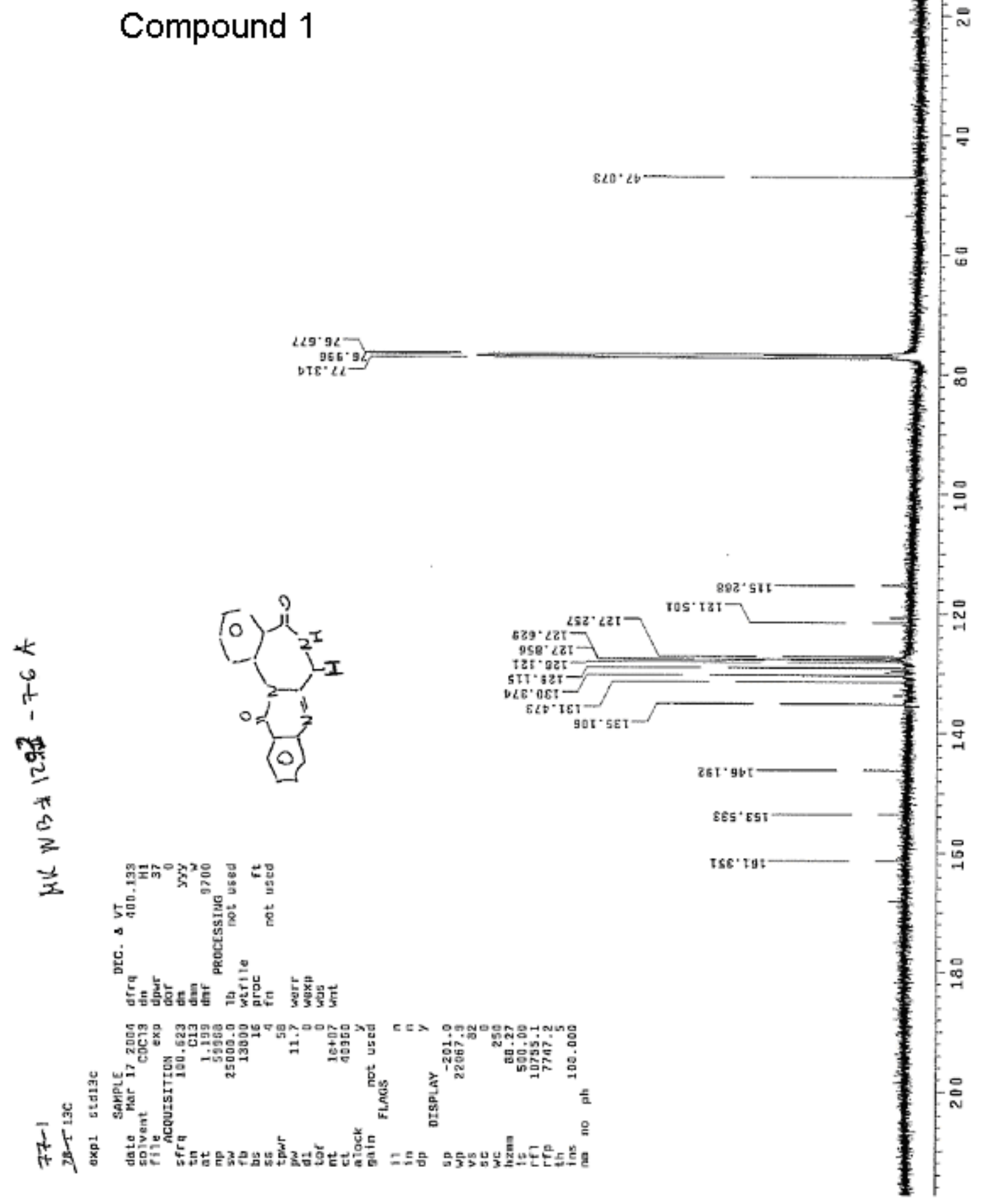




\section{SUPPORTING INFORMATION}

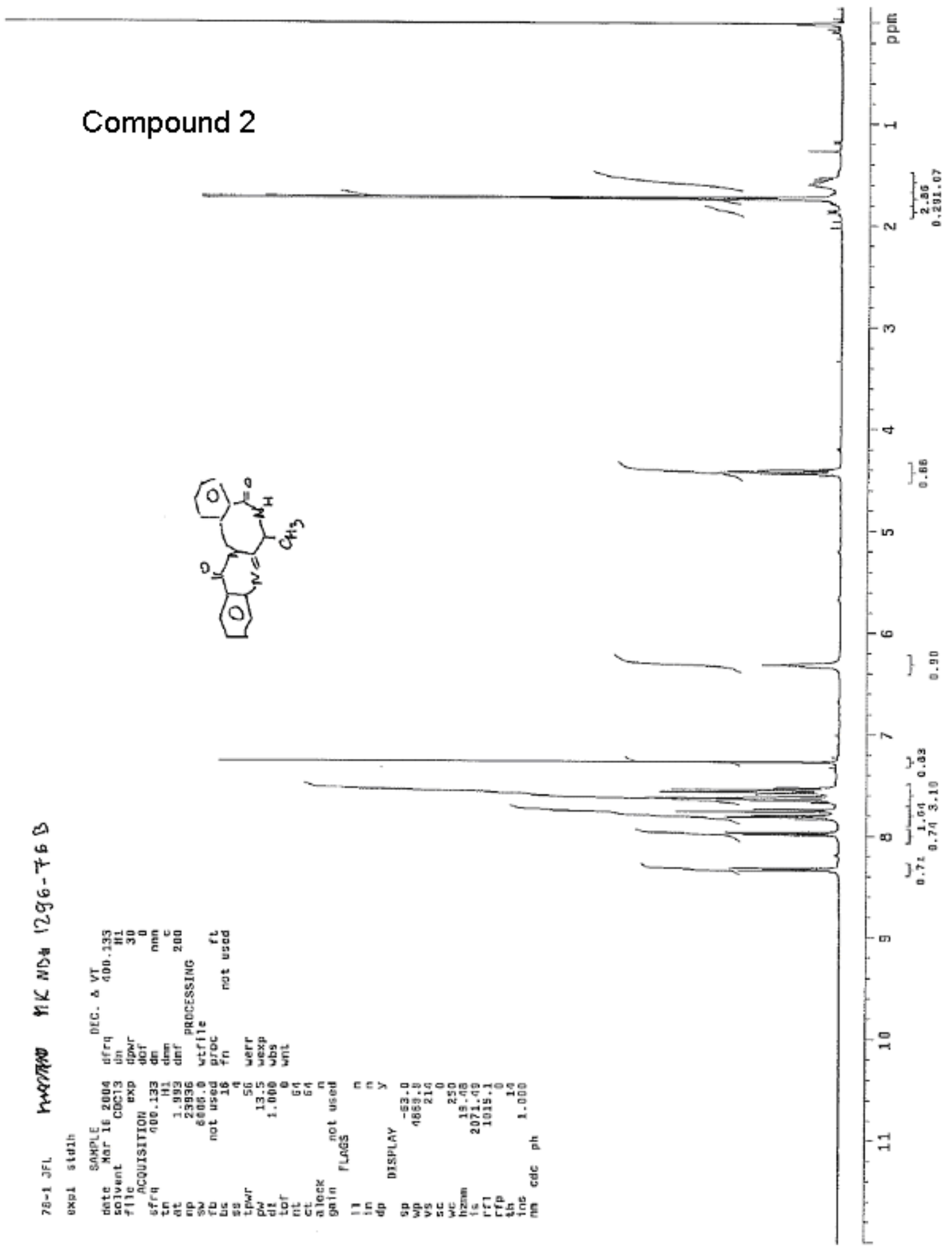




\section{SUPPORTING INFORMATION}

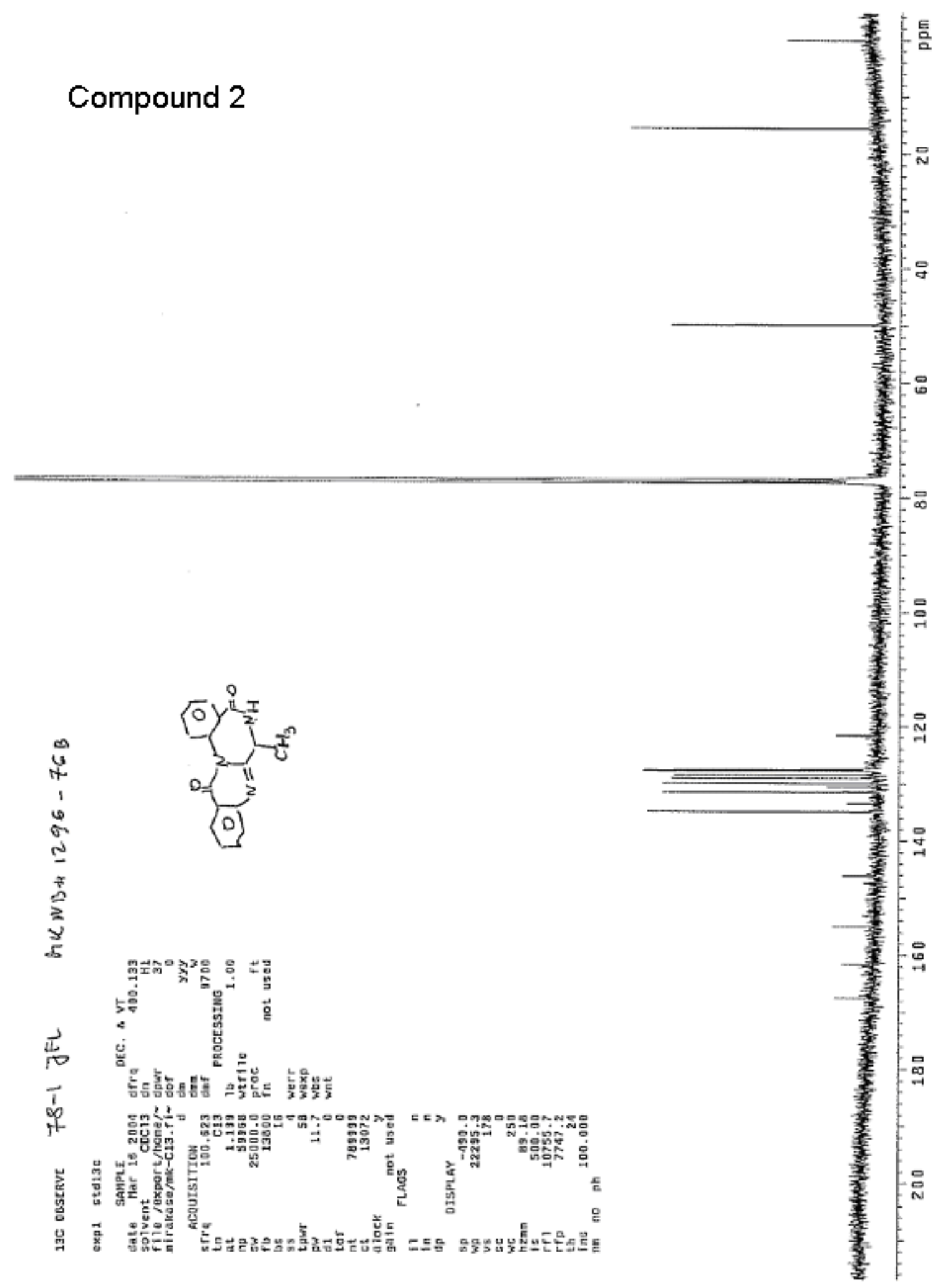




\section{SUPPORTING INFORMATION}

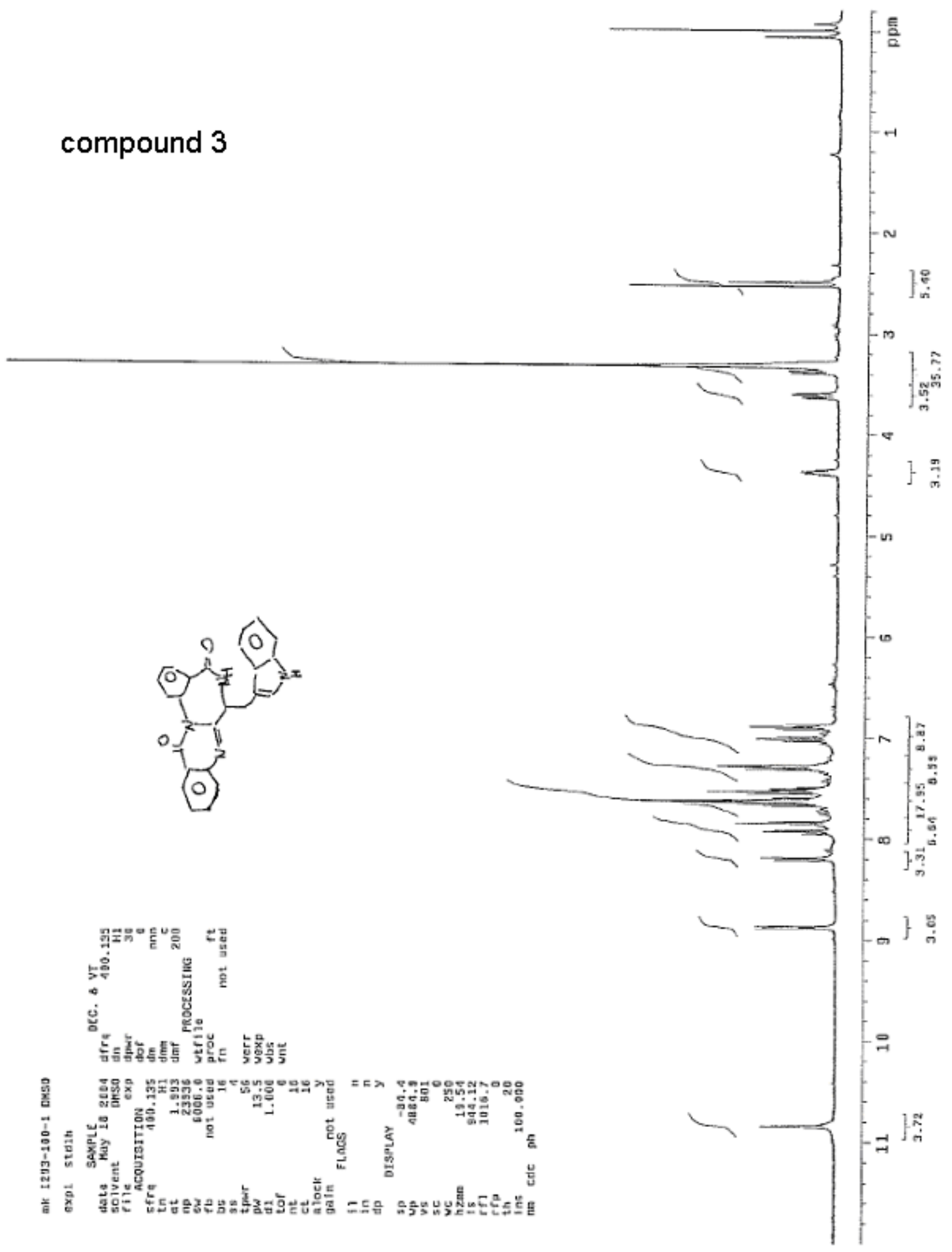




\section{SUPPORTING INFORMATION}

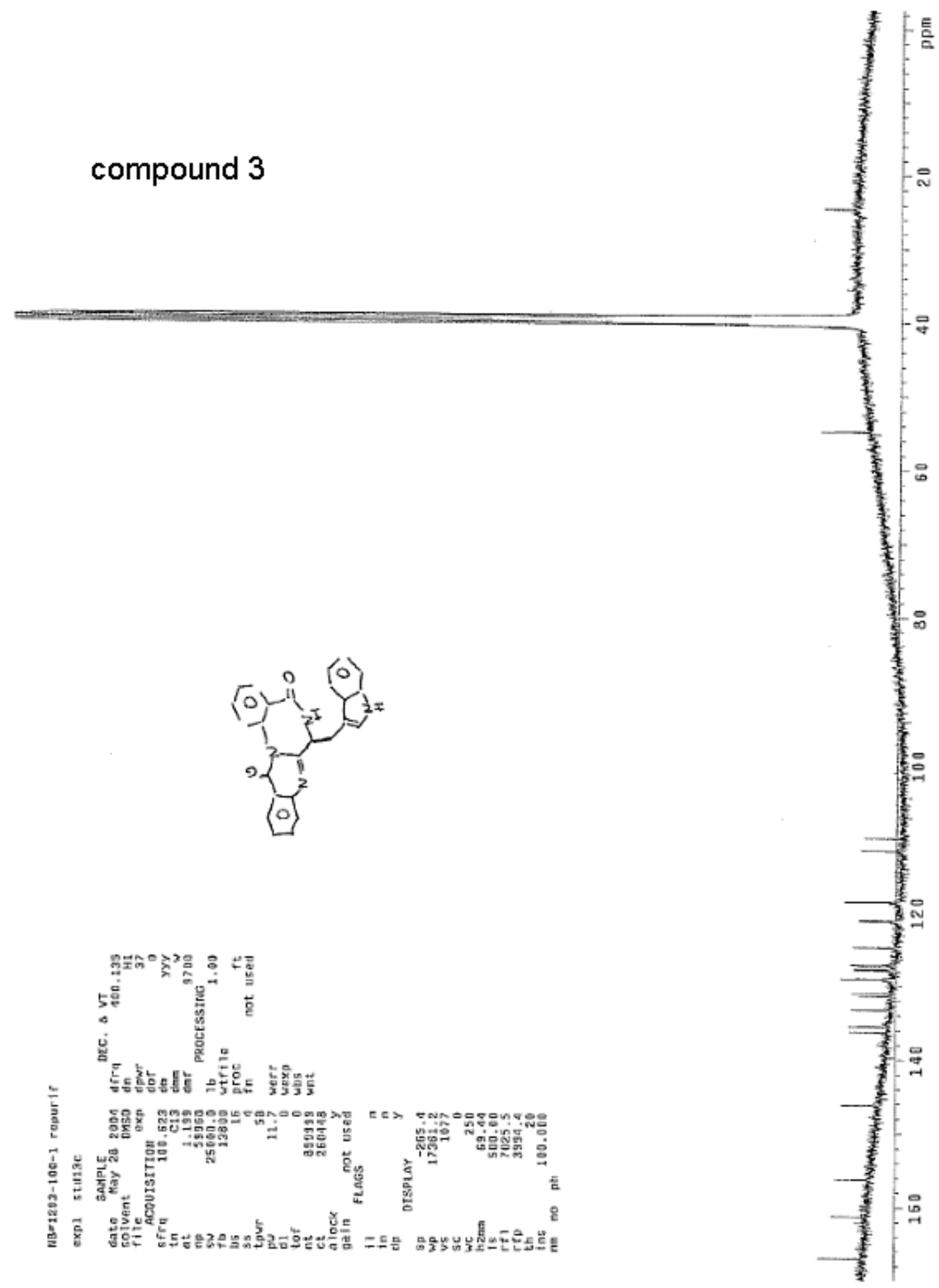




\section{SUPPORTING INFORMATION}

compound 15
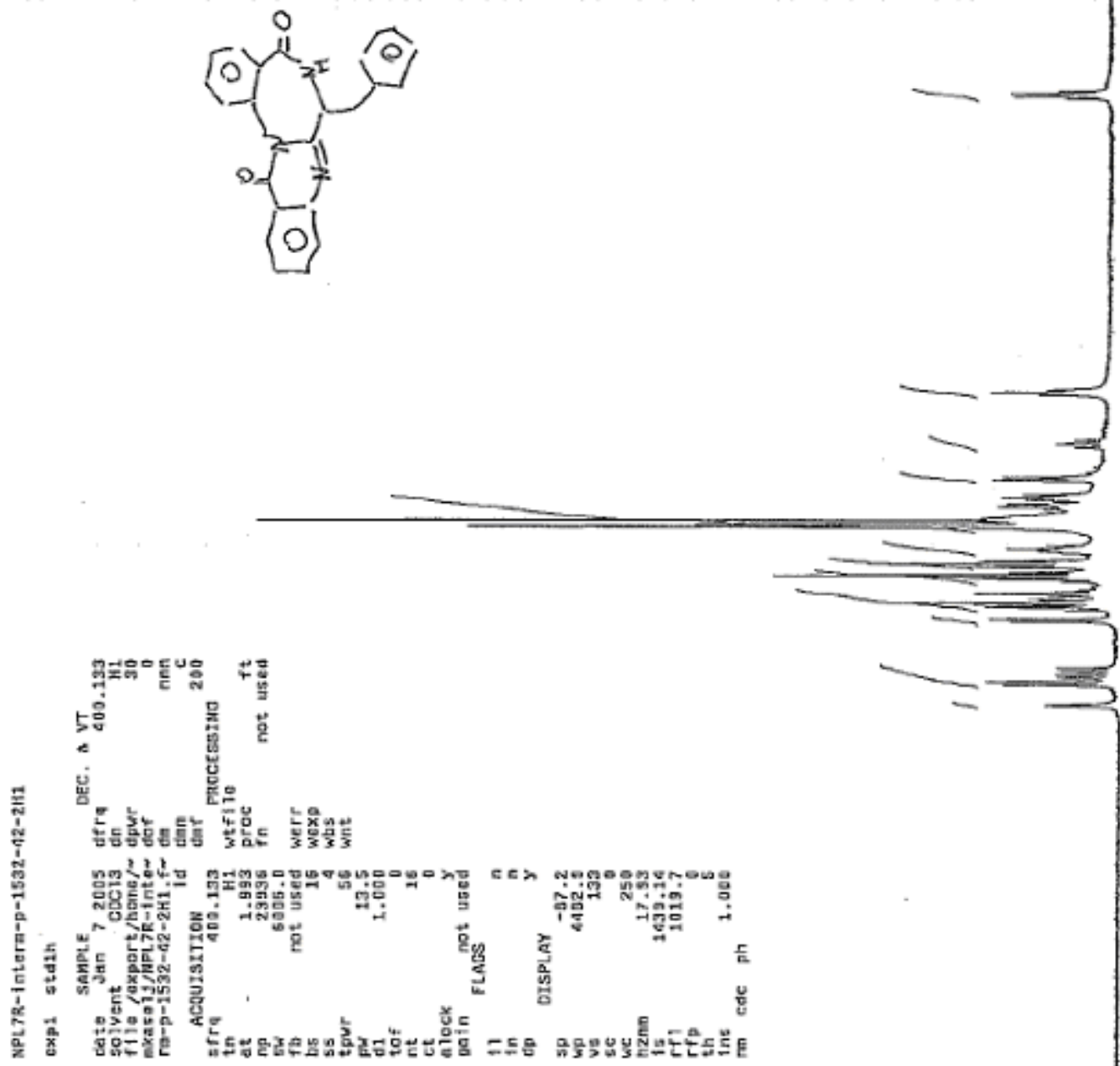

-

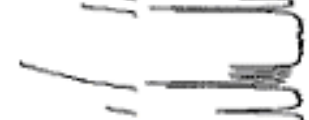




\section{SUPPORTING INFORMATION}

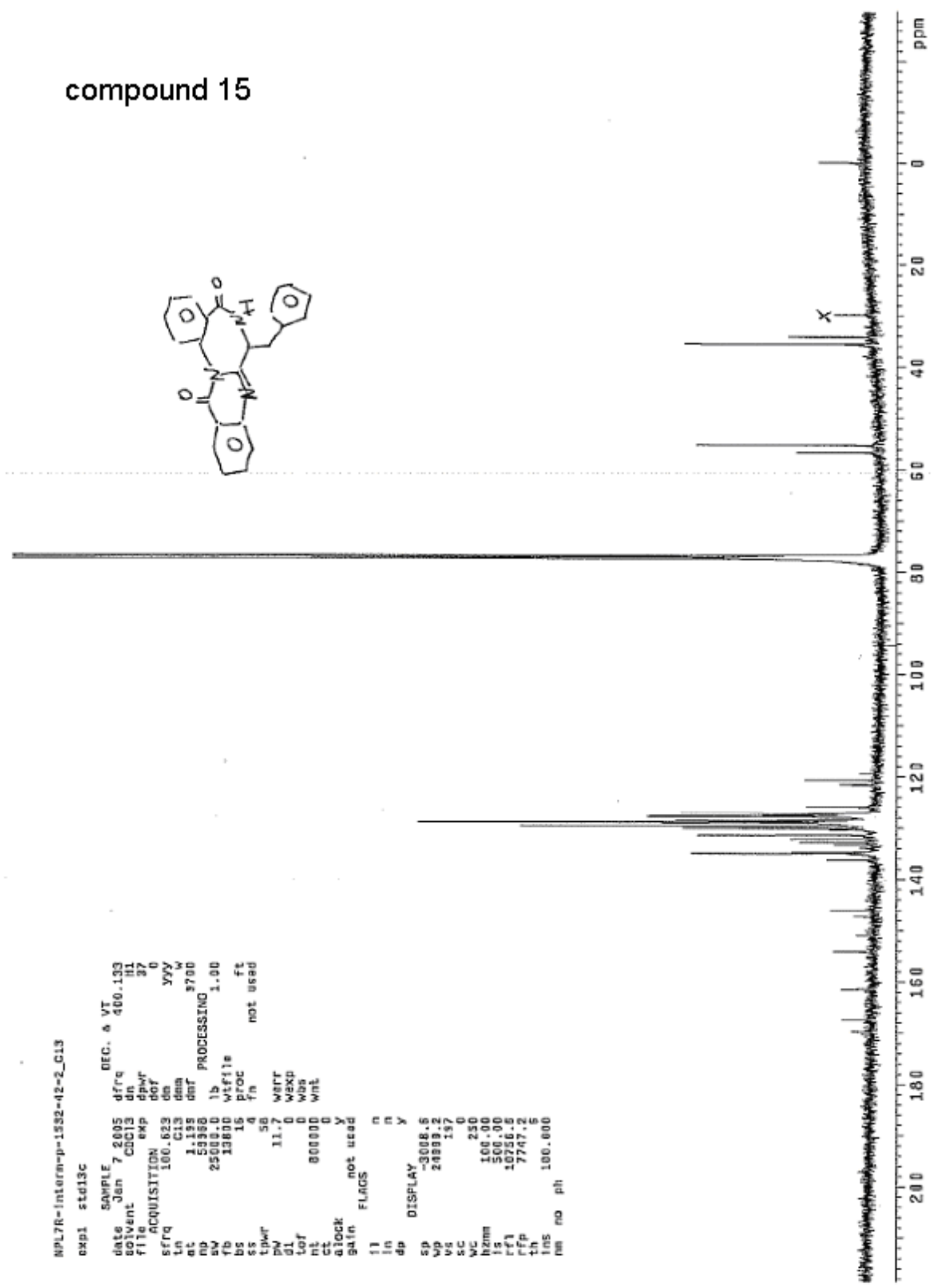




\section{SUPPORTING INFORMATION}

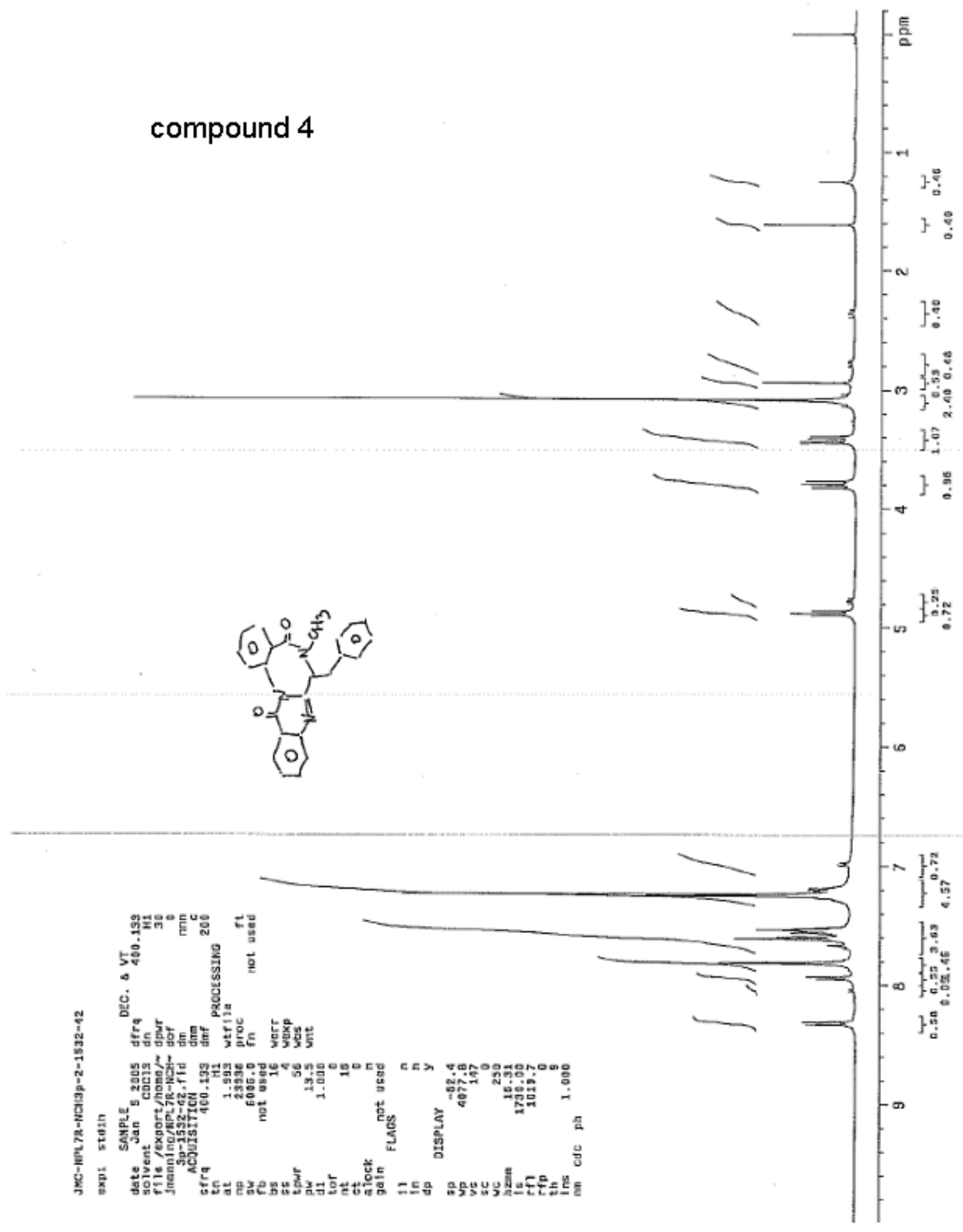




\section{SUPPORTING INFORMATION}

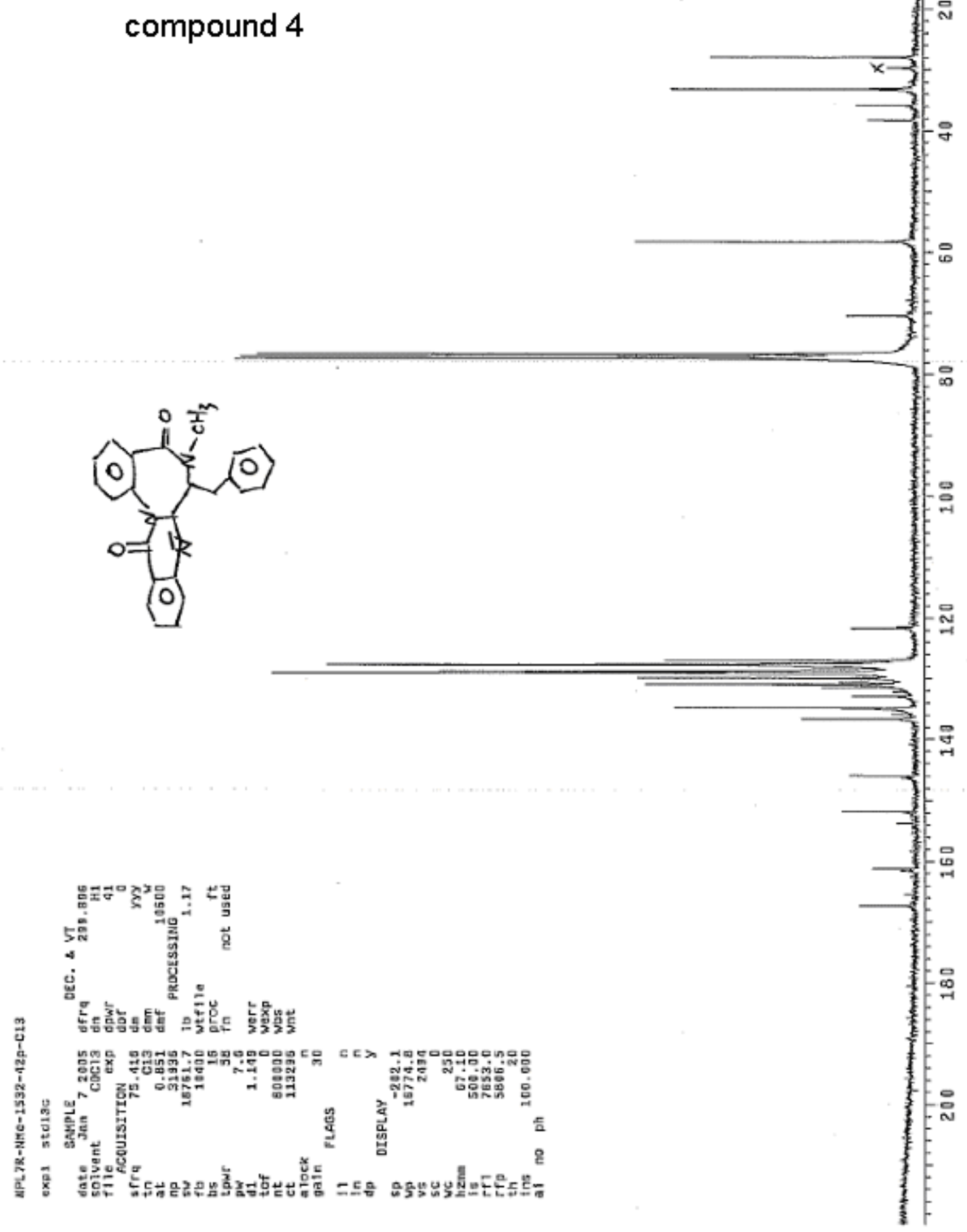




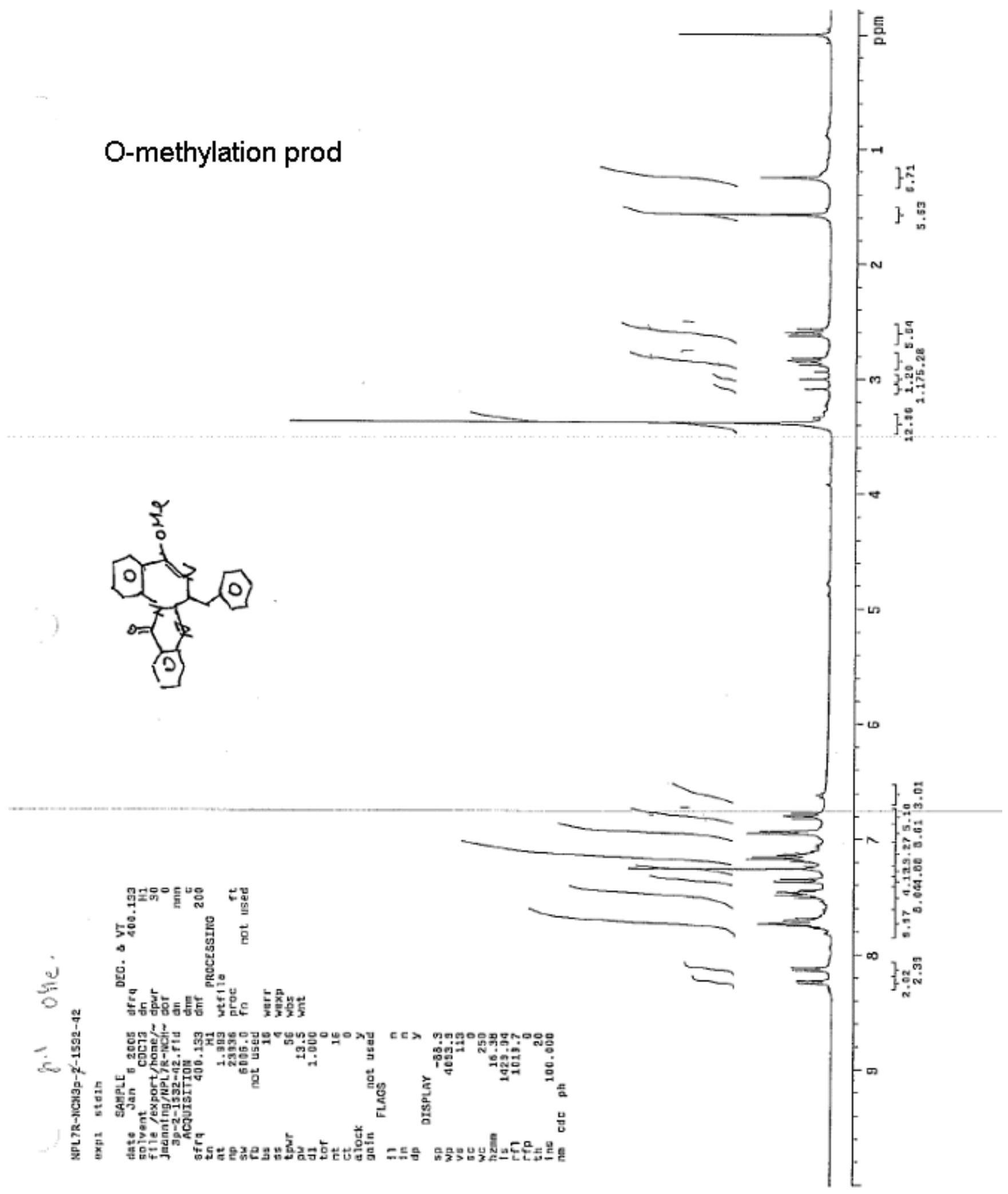




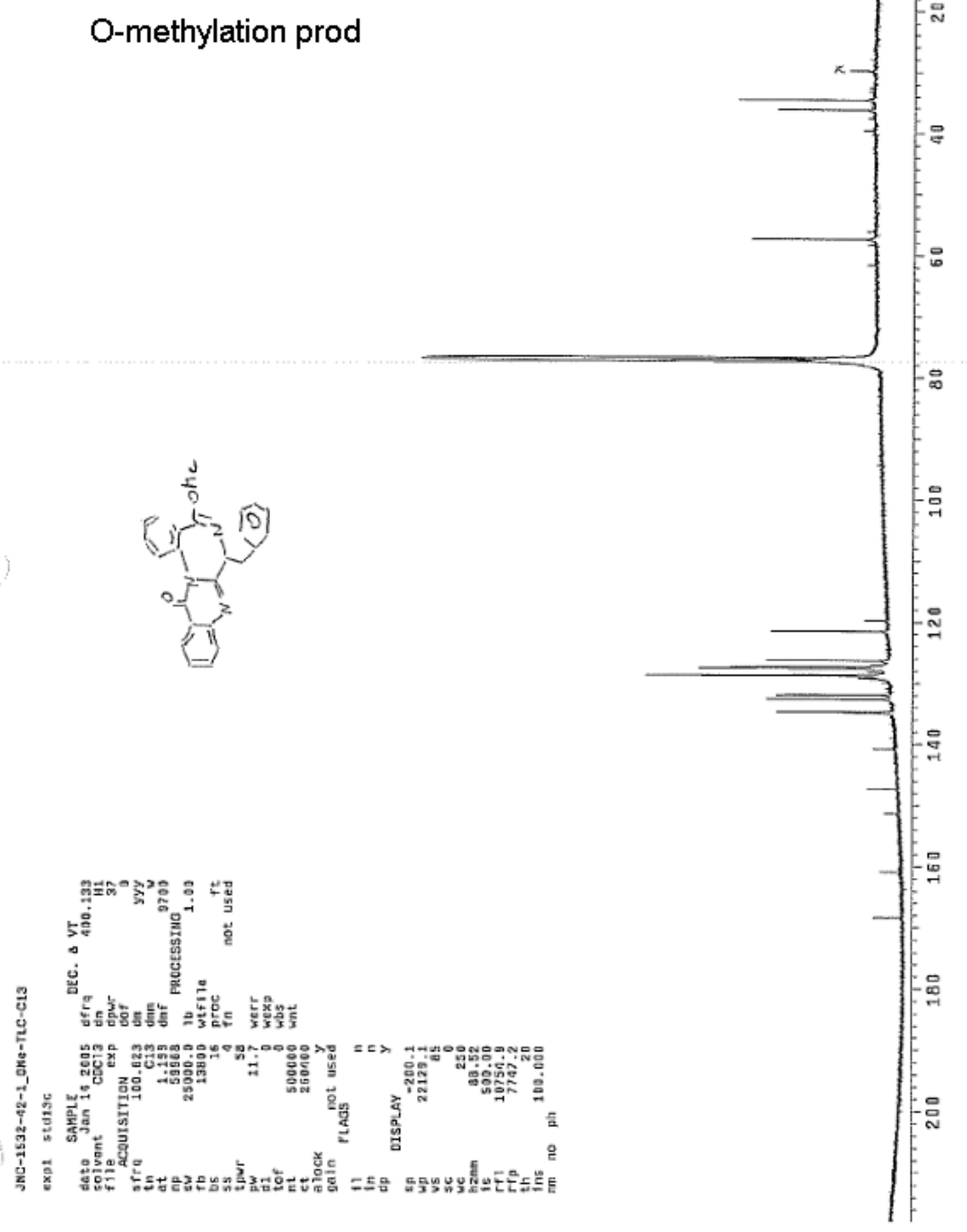




\section{SUPPORTING INFORMATION}

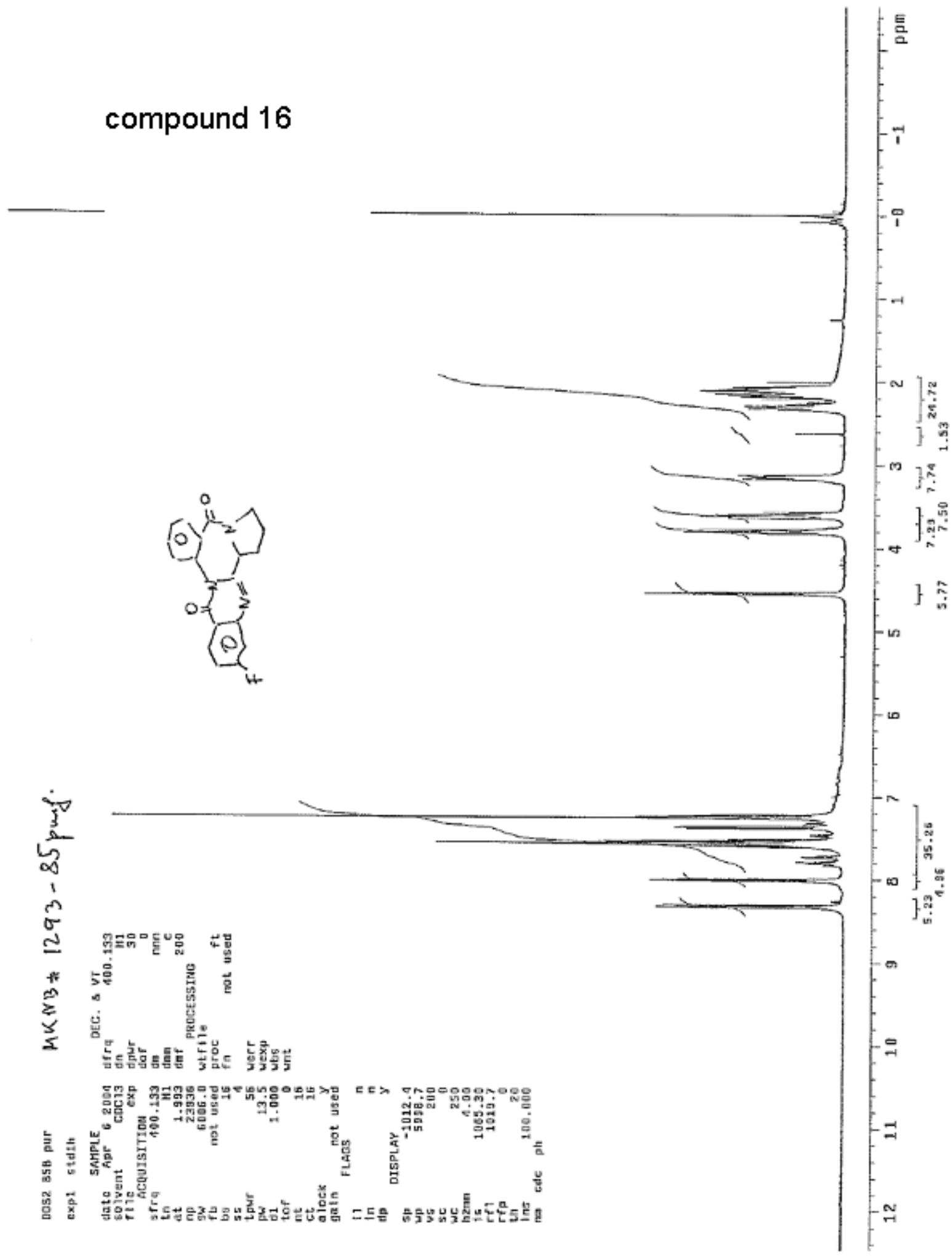




\section{SUPPORTING INFORMATION}

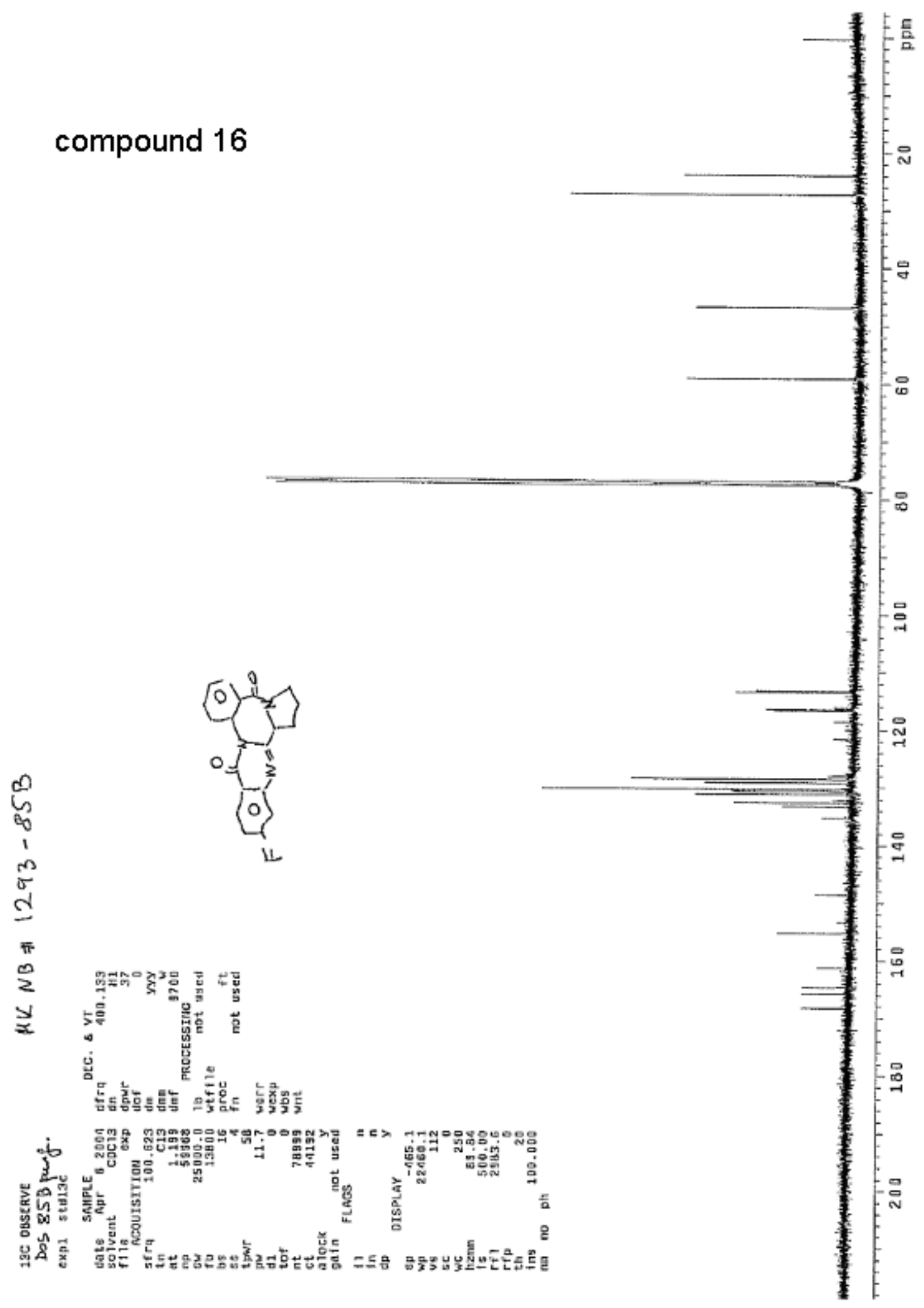




\section{SUPPORTING INFORMATION}

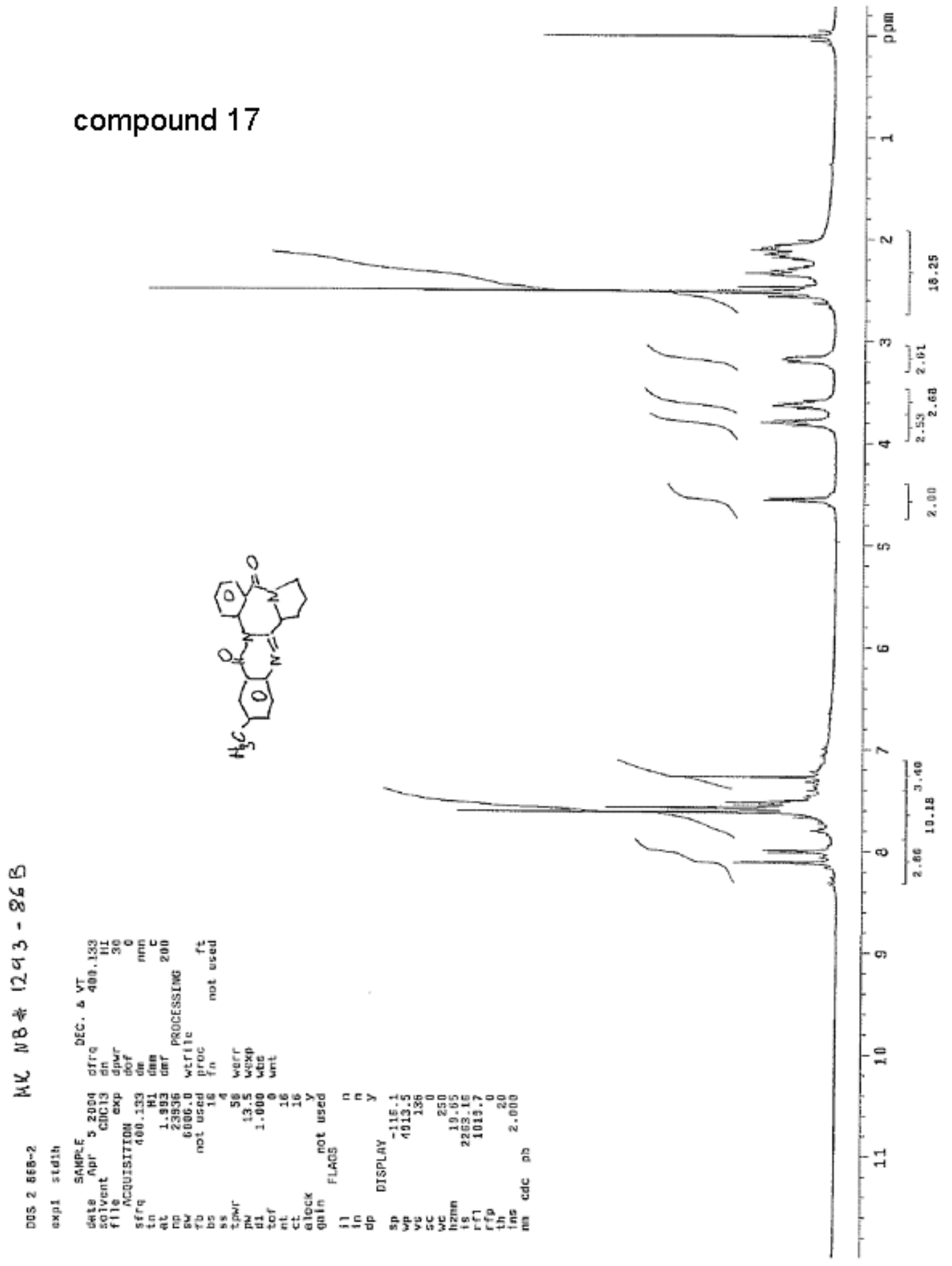



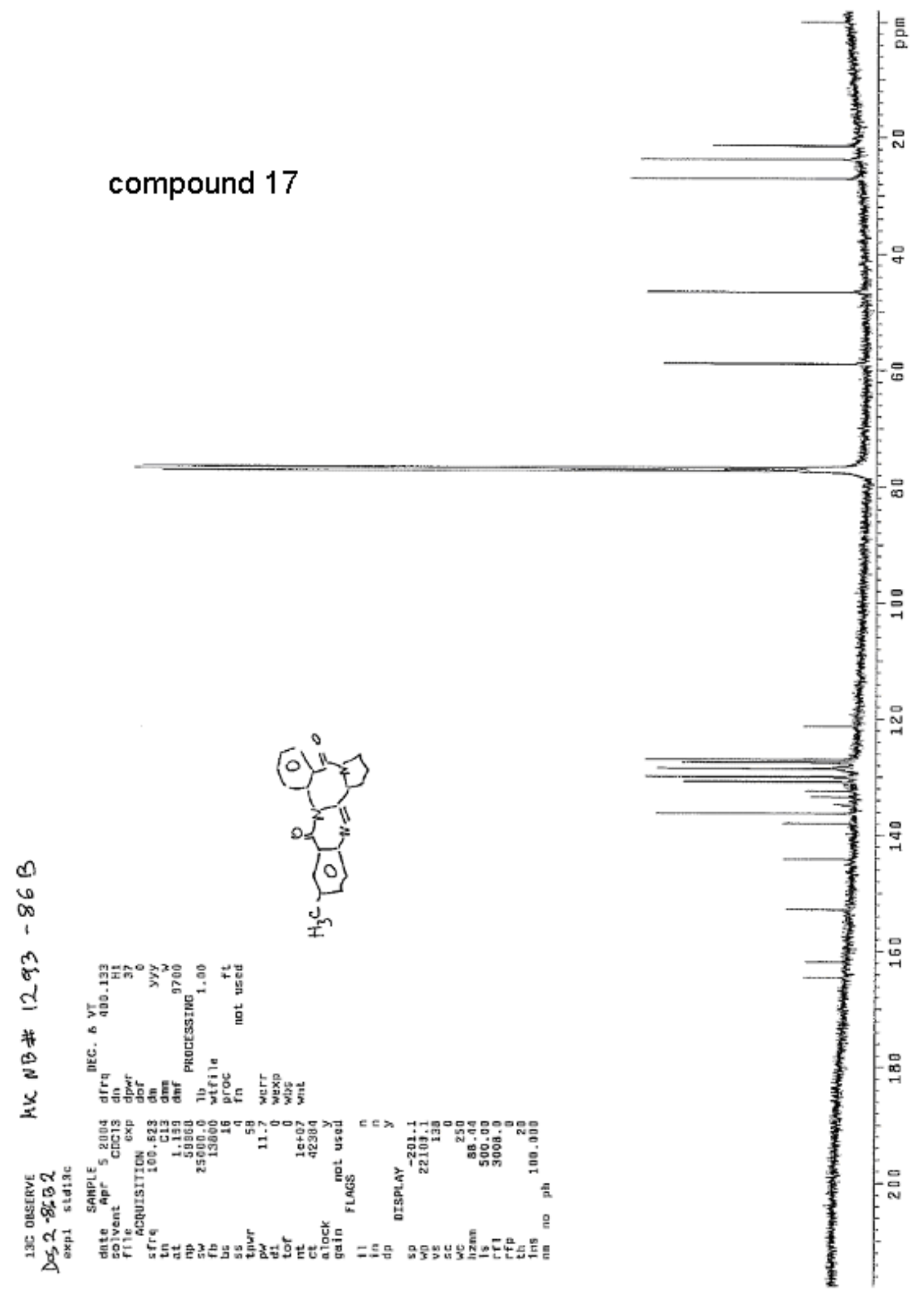